\title{
Diversidad y dinámica de un bosque subandino de altitud en la región norte de los Andes colombianos
}

\author{
Jesús Oswaldo Velásquez Restrepo, Nancy Lorena Maniguaje \& Álvaro Javier Duque \\ Universidad Nacional de Colombia Sede de Medellín. Calle 59A No 63-20 Medellín, Colombia. Apartado postal 3840 \\ Medellín,Colombia; jovelasq@unal.edu.co,nlmaniguaj@unal.edu.co,ajduque@unal.edu.co
}

Recibido 30-III-2011. Corregido 03-XI-2011. Aceptado 01-XII-2011.

\begin{abstract}
Diversity and dynamics of a high sub-Andean forest from Northern Andes, Colombia. The sub-Andean forests are characterized by a high biodiversity, but little is known about their natural dynamics. In order to generate new information, this study assessed two permanent plots of one hectare each, in the Northern Andean area of the Western Cordillera, Colombia. Methodology included the evaluation of diversity patterns, above ground biomass (AGB) dynamics, and mortality and recruitment rates. Besides, we used the Fisher's Alpha index to calculate species diversity. Forest dynamics and AGB were evaluated in both plots by means of three censuses carried out within a nine years period. In total, we found 1664 individuals with diameter at breast height $(\mathrm{DBH}) \geq 10 \mathrm{~cm}$ belonging to 222 species, 113 genera and 60 families. Mean species richness was of 156 species/ha and a mean Fisher's Alpha index of 56.2/ha. The mortality rate was $0.88 \%$ and recruitment was $1.16 \%$, which did not allow to lay any external effect of global warming or climate change on individual forest dynamics. However, the mean AGB was $243.44 \pm 9.82 \mathrm{t} / \mathrm{ha}$, with an annual average increase of $2.9 \mathrm{t} / \mathrm{ha}$, a higher value than the one reported in other studies of high sub-Andean forests, which suggests that equilibrium in terms of the AGB have not yet been reached. Besides, according to field observations, a recovery process, from a disturbance that occurred in the past, might be on his way. Rev. Biol. Trop. 60 (2): 943-952. Epub 2012 June 01.
\end{abstract}

Key words: above ground biomass, mortality, recruitment, climate change, lower montane forests.

Los bosques andinos han sido reconocidos como uno de los principales centros de diversidad del mundo (Myers et al. 2000, Brown \& Kappelle 2001). De acuerdo con Birdlife International, Boyla \& Estrada (2005), la región de los andes tropicales es la más rica y diversa del mundo desde un punto de vista biológico. En esta región, Perú, Colombia, Ecuador, Venezuela y Bolivia, han sido incluidos en la lista de los 17 países megadiversos, los cuales mantienen dentro de sus fronteras más de dos terceras partes de la riqueza biológica del planeta (Myers et al. 2000).

Según la mayoría de estudios, la riqueza y diversidad de especies decrece sistemáticamente a medida que se incrementa la altitud (Gentry 1995, Rosenzweig 1995); no obstante, algunos sugieren en cambio que la riqueza y diversidad de especies se magnifica en alturas intermedias como producto de la respuesta a restricciones fisiográficas asociadas con la topografía de las montañas (McCain 2004). En los bosques de montaña de los Andes (por encima de los $1800 \mathrm{msnm}$ ), la alta variación ambiental parece promover cambios abruptos en la estructura, composición y dinámica de la vegetación, incluso a escalas espaciales relativamente pequeñas (Homeier et al. 2010, López \& Duque 2010, Rangel-Ch et al. 2005).

A diferencia de lo que ocurre con la diversidad, los estudios de la dinámica natural de los bosques de montaña han recibido muy poca atención a pesar de la importancia que tienen para la conservación. Aspectos concretos como conocer las tasas de mortalidad y reclutamiento de especies y la biomasa de estos ecosistemas 
han sido muy poco estudiados (Sierra et al. 2007). Lo anterior, resulta especialmente cierto en Colombia, donde los bosques andinos han perdido aproximadamente un $70 \%$ de la cobertura original (Rodríguez et al. 2004, Chaves \& Santamaría 2006). Una de las zonas mejor estudiadas en cuanto a la dinámica de los bosques subandinos proviene del bosque de La Planada, en Colombia, donde se ha estimado una tasa de mortalidad anual de $3.22 \%( \pm 0.47)$ (Condit et al. 2006). A pesar de que se esperaría que las condiciones topográficas con altas pendientes promuevan el incremento de la mortalidad, las tasas arriba reportadas no difieren sustancialmente de las de otros bosques tropicales, como por ejemplo, los bosques secos de tierras bajas de la Isla de Barro Colorado (Condit et al. 2006, Cantillo \& Rangel-Ch 2008).

El cambio climático, además de las altas tasas de deforestación y cambio de uso del suelo, aparecen como amenazas para la sostenibilidad de los bosques tropicales de montaña (Colwell et al. 2008), a estos factores se suman la cacería, el comercio de especies silvestres y sus productos derivados. Los modelos actuales, sugieren que los bosques tropicales están experimentando cambios en su capacidad de almacenar y fijar carbono (ver Phillips et al. 1998), como producto del incremento en $\mathrm{CO}_{2}$ en la atmósfera (Eggleston et al. 2006). Sin embargo, la mayor parte de la discusión se ha enfocado en los ecosistemas de tierras bajas (Condit et al. 2004a, Phillips et al. 2004). En los bosques subandinos de La Planada en Colombia, por ejemplo, se ha reportado una tendencia al incremento en biomasa aérea de un $0.72 \%$ año, que equivale a 1.27ton/ha.año (Chave et al. 2008), lo cual está dentro de los rangos reportados para tierras bajas (Phillips et al. 2009). Aunque el aumento de la temperatura y concentración de $\mathrm{CO}_{2}$ favorecería teóricamente el crecimiento de los bosques montanos, la evidencia global (Chave et al. 2008), y la escasez de estudios de monitoreo en éste tipo de bosque tropical, no es aún suficiente para validar esta tendencia.

El presente estudio, fue llevado a cabo en bosques de montaña localizados en la Reserva Natural Farallones del Citará, en el municipio de Betania (Antioquia-Colombia), en dos parcelas permanentes de una hectárea, ubicadas en alturas entre los 2000 y $2200 \mathrm{msnm}$, y que fueron analizadas por un período de nueve años. El objetivo fue responder a las siguientes preguntas: 1) ¿Sigue el patrón de diversidad la tendencia encontrada para otros bosques subandinos de altitud?, 2) ¿La dinámica observada de individuos y biomasa indica alguna señal de respuesta al cambio climático?. Este estudio espera contribuir con el conocimiento de la estructura y funcionamiento de los bosques de alta montaña de la región neotropical con el fin de promover acciones para su conservación y restauración.

\section{MATERIALES Y MÉTODOS}

Área de estudio: La zona de estudio está ubicada en la vertiente oriental de la cordillera Occidental, dentro la región de los Farallones del Citará, en el municipio de Betania (Antioquia), en la vereda Pedral Arriba, predio Agualinda (Sánchez \& Velásquez 1997). La zona de vida se clasifica como Bosque muy Húmedo Montano Bajo (bmh-MB), denominada comúnmente como Bosque de Niebla (Espinal et al. 1977); las parcelas parecen pertenecer a una zona de transición entre el bosque muy húmedo y húmedo (Sánchez \& Velásquez 1997). La región de los Farallones del Citará se caracteriza por tener una topografía abrupta donde predominan relieves con fuertes pendientes y escarpados cerros con alturas hasta los 4000msnm en el Cerro de San Nicolás. La precipitación media anual en la región es de $2800 \mathrm{~mm}$, según datos tomados en la estación pluviográfica Ventanas ubicada a una altitud de $2850 \mathrm{msnm}$ en la misma cuenca hidrográfica del área de estudio, y se distribuye de forma bimodal con dos períodos extremadamente lluviosos intercalados con dos períodos con menor cantidad de precipitación. De esta manera, la zona posee un clima no estacional con ausencia de meses secos y sin presentarse déficit hídrico para las plantas (Sánchez \& Velásquez 1997). 
Desde el punto de vista geológico, en estas zonas se presentan rocas sedimentarias datadas como del período Juratriásico, compuestas por arcillositas pizarrosas, capas de areniscas y algunos conglomerados. Se han determinado algunos sedimentos tales como la formación Penderisco del Grupo Cañasgordas y de origen cretácico, y las graníticas, que fueron asociadas al batolito de farallones del Mioceno superior, se ha señalado también la presencia de un pequeño sector de rocas volcánicas jóvenes, posiblemente del Plioceno (Parra 1997). En lo que se refiere a los suelos, para la zona de los Farallones del Citará se reconocen como superficiales a moderadamente profundos, ácidos y de fertilidad baja a moderada y pendientes por encima del $75 \%$ que a veces se entremezclan con afloramientos rocosos (Vanegas 1997). Las principales clases son: Udorthents, Dystrudepts y Hapludands.

Descripción general de la vegetación: Los ecosistemas boscosos de la Región de los Farallones del Citará corresponden a los denominados bosques montanos, los cuales no contienen un tipo específico de vegetación sino que se encuentran grandes mezclas de especies conformando ecosistemas de los cuales muy poco queda de ellos en Colombia y los que quedan están seriamente amenazados (Sánchez \& Velásquez 1997).

Censos de vegetación: Los datos sobre composición florística y de la vegetación se tomaron en dos parcelas ubicadas a una altura de $2030 \mathrm{msnm}$ (altura de la línea base) (544'545" N - 7601'100” W) (Sánchez \& Velásquez 1997). En la zona de estudio se demarcó una parcela de 500x500m (25ha). La línea base se dividió en 25 transectos de $20 \mathrm{~m}$ cada uno, los cuales se identificaron con letras (desde la A hasta la Y). Las parcelas en estudio fueron de 20x500m (una ha), en disposición perpendicular a la línea base, la primera se ubicó entre los 80 y 100m (llamada parcela E, en adelante) y la segunda entre los 400 y $420 \mathrm{~m}$ (llamada parcela $\mathrm{U}$, en adelante). Cada una de las parcelas fueron divididas en 25 cuadrantes de 20x20m, y estos a su vez se dividieron en sub-cuadrantes de 10x10m, marcados de uno a cuatro en el sentido de las manecillas del reloj. En cada uno de ellos se midieron, marcaron y mapearon todos los árboles con diámetro normal $\geq 10 \mathrm{~cm}$ (DAP). La colección botánica se realizó para todos los árboles, se tomaron de tres a cinco ejemplares para la colección general del herbario Gabriel Gutiérrez Medel (MEDEL). La identificación se realizó por comparación en los herbarios MEDEL, Universidad de Antioquia (HUA), Jardín Botánico de Medellín Joaquín Antonio Uribe (JAUM) y el Herbario Nacional Colombiano (COL) de la Universidad Nacional de Colombia. Adicionalmente, se utilizaron claves y la colaboración de especialistas.

El establecimiento de las parcelas se inició en el año 1996 (censo uno, en adelante C1), dentro del proyecto Biopacífico (Sánchez \& Velásquez 1997). En los años 1998 (C2) y 2000 (C3), la Universidad Nacional de Colombia sede Medellín en convenio con Corantioquia, realizaron una nueva medición de las parcelas. La cuarta, y última medición de los árboles se realizó en el año 2005 (C4), durante las labores de mantenimiento de las parcelas. Así, los periodos de medición serán 1996-1998 se citará como P1, 1998-2000 como P2 y 2000-2005 como P3. Sin embargo, es importante anotar que en los sitios para el establecimiento de las parcelas se buscó un área donde se evidenciara una estructura y estado de conservación del bosque lo más ideal o representativo de los ecosistemas alto andinos de la región.

Para el análisis de datos se emplearon las siguientes ecuaciones e índices:

Diversidad: La diversidad de plantas se estimó en las parcelas mediante el uso del índice Alfa de Fisher (Condit et al. 1996), el cual se define por:

$$
S=\alpha \ln \left(1+\left(\frac{N}{\alpha}\right)\right)
$$

donde $\mathrm{S}$ es el número de especies en una muestra de $N$ individuos y $\alpha$ (Alfa de Fisher) 
es una constante independiente de $N$. Se halló el índice para cada parcela y el promedio por hectárea utilizando los respectivos promedios de número de especies e individuos. El alfa de Fisher se calculó utilizando el método de Newton (Condit et al. 1996).

Dinámica: Para el cálculo de las tasas de mortalidad $(m)$ y reclutamiento $(r)$ se utilizaron las ecuaciones estándar:

$$
m=\frac{\left[\ln \left(\frac{N_{0}}{S}\right)\right]}{T}, \quad r=\frac{\left[\ln \left(\frac{N_{t}}{S}\right)\right]}{T}
$$

donde $T$ es el intervalo de tiempo entre censos, $N_{0}$ y $N_{t}$ son los tamaños poblacionales en el censo cero y en el censo $t$ respectivamente, y $S$ es el número de sobrevivientes en el tiempo $t$.

Además, se halló el crecimiento poblacional como,

$$
\lambda=\frac{\left[\ln \left(\frac{N_{t}}{N_{0}}\right)\right]}{T}
$$

es decir, $\lambda=r-m$.

Biomasa: La biomasa de todos los individuos se calculó usando la ecuación $\ln \mathrm{B}=-2.286+2.471 \ln \mathrm{D}\left(\mathrm{n}=140, \mathrm{R}^{2}=97.9 \%\right)$, donde $\mathrm{B}$ es la biomasa en $\mathrm{kg}$ y $\mathrm{D}$ es el diámetro a la altura del pecho (DAP) en cm (Zapata et al. 2003). Aunque esta ecuación proviene de un tipo de bosque diferente, donde existe un clima estacional y una mayor temperatura, la cercanía geográfica del sitio donde fue elaborada y su condición de bosque de montaña, fueron los criterios seleccionados para su elección ante la inexistencia de modelos alométricos de biomasa específicos para bosques de alta montaña. La biomasa total para cada parcela y por periodos de censo, se calculó como la suma de la biomasa de todos los árboles vivos. Se estimó el balance de la dinámica de la biomasa aérea del bosque $(\triangle B A)$ como: $\triangle B A=\Delta S+I-$ $M$ donde $\triangle S$ es el incremento de la biomasa de las plantas sobrevivientes entre censos; $I$ es la biomasa de los árboles que ingresaron y, $M$ es la biomasa de los árboles que murieron entre dos mediciones.

\section{RESULTADOS}

Diversidad: En el censo correspondiente al año 2005 se registró un total de 1664 individuos con $\mathrm{DAP} \geq 10 \mathrm{~cm}$, distribuidos en 222 especies, 113 géneros y 60 familias. En el cuadro 1 se observa el número de individuos, especies, géneros y familias en cada uno de los censos

\section{CUADRO 1}

Número de individuos observado en cada uno de los años de medición en las dos parcelas de 1 ha establecidas

TABLE 1

\begin{tabular}{|c|c|c|c|c|}
\hline Parcela & Censo & N. ${ }^{\circ}$ familias & N. ${ }^{\circ}$ especies & N. ${ }^{\circ}$ individuos \\
\hline \multirow{4}{*}{$\mathrm{E}$} & 1996 & 50 & 128 & 881 \\
\hline & 1998 & 50 & 127 & 880 \\
\hline & 2000 & 50 & 127 & 873 \\
\hline & 2005 & 49 & 129 & 902 \\
\hline \multirow{4}{*}{$\mathrm{U}$} & 1996 & 56 & 173 & 737 \\
\hline & 1998 & 54 & 168 & 749 \\
\hline & 2000 & 53 & 166 & 728 \\
\hline & 2005 & 52 & 175 & 762 \\
\hline \multirow{4}{*}{ Promedio por ha } & 1996 & 53 & 150,5 & 809 \\
\hline & 1998 & 52 & 147.5 & 814.5 \\
\hline & 2000 & 51.5 & 146.5 & 800.5 \\
\hline & 2005 & 50.5 & 152 & 832 \\
\hline
\end{tabular}

Number of individuals recorded by census in each one of the two plots established 
y parcelas. Las especies más representativas en el último censo fueron: Alfaroa colombiana (11.19\% del total de los individuos), Croton smithianus (3.97\%), Chrysochlamys colombiana (2.71\%), Calophyllum brasiliense (2.11\%), Pouteria lucuma $(2.05 \%)$ y Ocotea costulata (2.04\%). En el sitio de estudio las familias con mayor número de especies fueron: Lauraceae (31 especies), Rubiaceae (17), Euphorbiaceae (14), Melastomataceae (14) y Clusiaceae (9). El valor del alfa de Fisher, en el año 2005 para la parcela $E$ fue de 41.2 y para la $U$ fue de 71.11, para las dos hectáreas el índice fue de 68.82. En promedio el alfa de Fisher por hectárea fue de 56.2 (Cuadro 1).

Dinámica: Las tasas de mortalidad $(\mathrm{m})$ y reclutamiento $(r)$ durante los cuatro censos mostraron valores entre $1.21-1.63 \%$ y $0.44-$ $2.10 \%$, respectivamente. En el periodo global 1996-2005 la tasa de mortalidad fue de $0.88 \%$ y la de reclutamiento $1.16 \%$. El crecimiento poblacional para el periodo total fue $0.28 \%$; entre censos fue $0.28 \%$ para 1996 $1998,0.77 \%$ para $1998-2000$ y $0.71 \%$ para 2000-2005 (Fig. 1).

Biomasa: La biomasa aérea total en promedio para el sitio de estudio fue de 243.44 $\pm 9.82 \mathrm{t} /$ ha (IC 95\%) para el periodo entre 1996-2005, con valores entre $229.05-266.25 \mathrm{t} / \mathrm{ha}$. El valor más alto se presentó en el año 2005 con una biomasa aérea total de $260.44 t$ /ha y el más bajo fue de 231.33t/ha en el primer censo (Fig. 2).

El incremento anual promedio en la biomasa aérea fue de 2.9t/ha (1.2\%); el mayor valor se presentó en el primer periodo con un incremento de 5.9t/ha.año y en el periodo entre 1998-2000 se presentó una pérdida en biomasa de 2.14t/ha.año (Fig. 3).

\section{DISCUSIÓN}

Diversidad: Los resultados de este estudio, difieren significativamente de lo esperado en cuanto a la riqueza de especies y diversidad en ecosistemas de alta montaña, ya que igualan e incluso superan la riqueza de especies reportada para ecosistemas húmedos de tierras bajas (Condit et al. 2004b) y para bosques montanos neotropicales (Cascante-Marín et al. 2011). Cuando se reportan valores de hasta $175 \mathrm{spp} / \mathrm{ha}$ en bosques a más de $2000 \mathrm{msnm}$, aparece una alta incertidumbre en la posibilidad de identificar el mecanismo fundamental que promueve semejante diversidad. Cifras tan altas en el número de especies en bosques de tierras altas, podrían conducir a especular que se deben en buena parte a errores en la identificación, posiblemente debido a un menor conocimiento

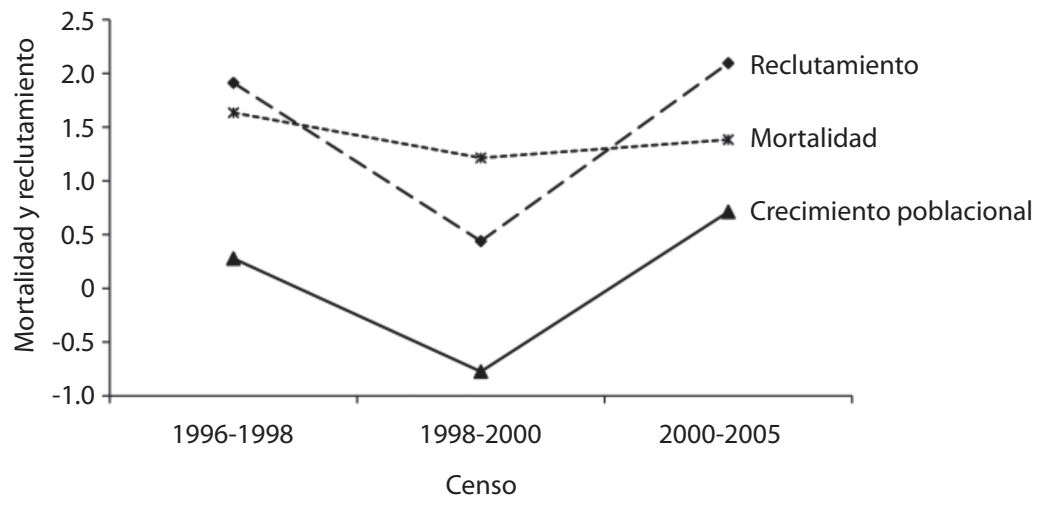

Fig. 1. Tasas de mortalidad, reclutamiento e individuos supervivientes (\%), durante cada uno de los periodos de medición. Mortalidad y reclutamiento (\%)

Fig. 1. Rates of mortality, recruitment and survivorships (\%) during each census. The dashed line represents mortality, the discontinuous line represents recruitment and the continuous line represents the growth population rate. 


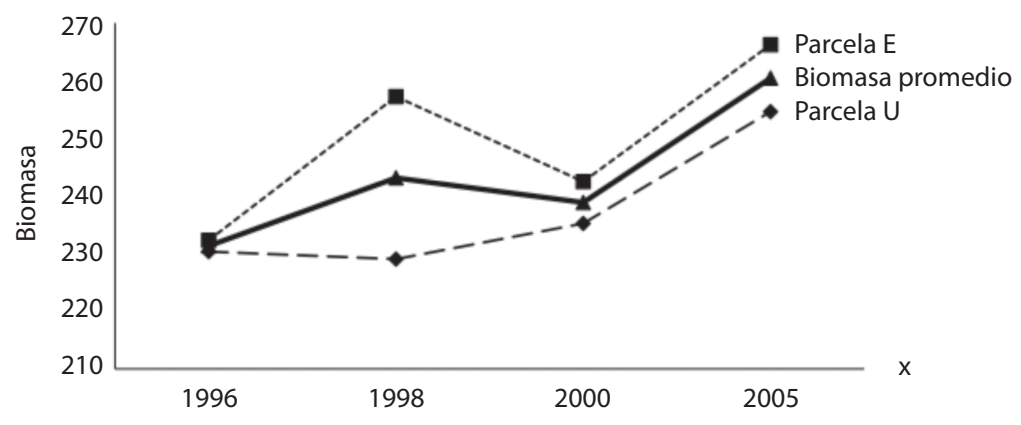

Fig. 2. Biomasa aérea total en t/ha en cada uno de los censos y parcelas, y promedio en los Farallones del Citará, Municipio de Betania, Antioquia.

Fig. 2. Above ground biomass (t/ha) in each census and plot. The continuous line represents the average by year. Upper dashed line represents plot $\mathrm{E}$, and the discontinuous line represents plot $\mathrm{U}$.

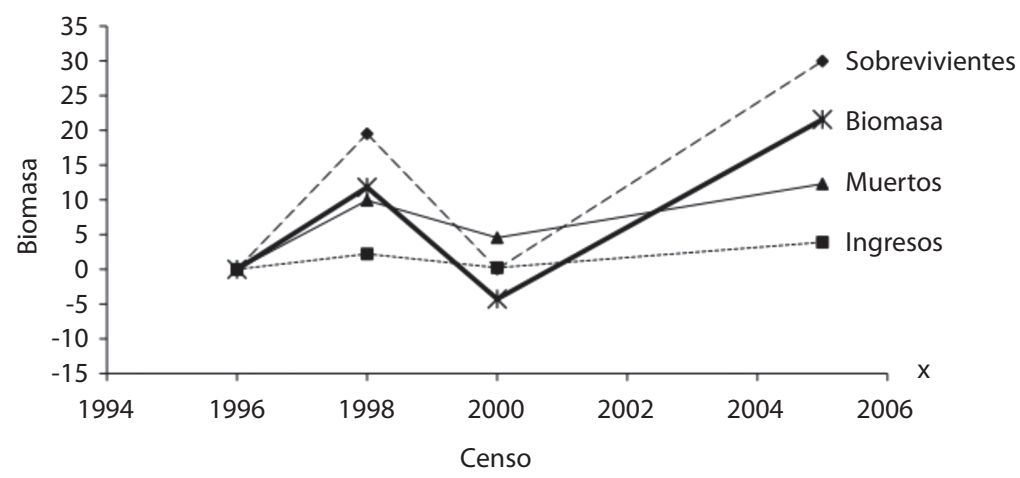

Fig. 3. Balance Promedio Neto (t/ha) de la biomasa aérea total, de supervivientes, de ingresos e individuos muertos, entre periodos de medición, para las dos parcelas de 1-ha monitoreadas en los Farallones del Citará, Antioquia.

Fig. 3. Average net above ground biomass balance (t/ha) in the two sub-Andean forests studied.

de la flora de estas zonas que la de los bosques de tierras bajas. Sin embargo, la taxonomía de estas plantas ha sido controlada por los mismos curadores desde la primera medición, incluyendo las otras dos re-mediciones, lo cual disminuye significativamente el margen de error en la identificación. Nótese que los porcentajes de especies indeterminadas estuvieron entre $25-30 \%$, el cual se considera dentro de los rangos esperados para este tipo de estudios (Ruokolainen et al. 2002), lo cual le resta a su vez incertidumbre a este aspecto. Por este motivo, la más plausible explicación para tan alta riqueza de especies se puede adjudicar a la posición biogeográfica de estos bosques localizados en el puente conector entre América del Sur y América Central, que se caracterizan por un alto endemismo y diversidad (Gentry 1982, López \& Duque 2010). De hecho, estudios de mamíferos y aves realizados en esta misma zona, (Cuadros 1997) han, a su vez, reportado registros y valores de diversidad en la misma línea de los reportados en este estudio para plantas leñosas.

Dinámica: La dinámica natural de este bosque, según sus tasas de mortalidad y reclutamiento, aunque presentó un incremento neto 
poblacional de $0.28 \%$ año, se puede considerar incluso como bajo para ecosistemas localizados en zonas de pendientes de hasta $68^{\circ}$. Una mortalidad de individuos de un $0.88 \%$ en promedio anual, se encuentra por debajo de la media reportada para bosques tropicales (Condit et al. 2006) y para bosques premontanos para los cuales se reportan valores de $2.3 \%$ (CascanteMarín et al. 2011). De esta manera, los resultados de este estudio, no permiten identificar ningún efecto externo proveniente de la variación climática como determinante de cambios en la densidad y patrón de reclutamiento de individuos arbóreos en este bosque subandino alto. Las razones para explicar la dinámica reportada, proviene de la posible recuperación del bosque luego de un disturbio natural a escala menor, lo cual no permite inferir efectos de cambio climático en este ecosistema. Sin embargo, cuando se analizó la dinámica del bosque en función de la biomasa aérea, los resultados fueron lo contrario.

Biomasa: En este bosque, la cantidad de biomasa aérea almacenada $(243.44 \pm 9.82 \mathrm{t} / \mathrm{ha})$ tuvo un comportamiento similar a lo reportado para la diversidad de especies, siendo en términos generales extremadamente alta. Cuando comparamos este valor con el de otros bosques en la misma zona de estudio, se evidencia que el bosque de Los Farallones dobla la biomasa esperada de bosques a una altitud similar (Yepes et al. 2011, com. pers.), aunque asemeja lo reportado para bosques premontanos en la misma cordillera central (Sierra et al. 2007). Una posible explicación a este fenómeno podría deberse a que los sitios donde se establecieron las parcelas fueron seleccionados siguiendo criterios que buscaban encontrar un bosque maduro y en buenas condiciones de conservación (majestic forest effect, sensu Phillips et al. 2004), el cual sólo es posible de encontrar en ciertas áreas aisladas. No obstante, su localización en zonas distantes de poblados y de difícil acceso, lo cual ha evitado que estos bosques se hayan visto sometidos a disturbios provenientes del aprovechamiento maderero, podría ser una buena explicación de la diferencia con respecto a otras áreas localizadas dentro de la misma franja ambiental. Datos recientes de la distribución de la biomasa en Colombia sugieren que los bosques húmedos montanos albergan en promedio por unidad de área una biomasa similar a los bosques húmedos de tierra bajas, lo cual sustenta los registros aquí reportados (Sánchez \& Velásquez 1997).

El incremento en biomasa reportado para este bosque (2.9ton/ha.año), dobla en magnitud lo reportado para otros bosques subandinos como por ejemplo el de La Planada y puede considerarse dentro del límite superior reportado para bosques tropicales de tierras bajas, superando en porcentaje (1.2\%) casi todos los registros recientes para bosques tropicales (Chave et al. 2008); no obstante, valores similares en incremento de biomasa han sido reportados para bosques amazónicos (Phillips et al. 2004). Sin embargo, aunque según lo observado en el campo este bosque simplemente parece encontrarse en una dinámica de recuperación hacia el equilibrio luego del desplome de grandes árboles en el periodo 1998-2000, el promedio anual del incremento en biomasa reportado (2.9ton/ha.año), resulta totalmente opuesto a la tendencia de la densidad. Bajo este escenario, podría argumentarse que factores externos como el calentamiento global si podrían estar influenciando el incremento en biomasa de este bosque, lo cual hace este reporte aún más extremo y difícil de explicar por las limitaciones climáticas, topográficas y fisiológicas de las especies para aumentar la capacidad de carga del sistema.

En síntesis, los resultados de este estudio no parecen evidenciar un posible efecto proveniente del calentamiento global sobre la dinámica y capacidad de almacenamiento del carbono de los bosques de alta montaña (Phillips et al. 2004). No obstante, los datos aquí presentados simplemente resaltan el alto desconocimiento que se tiene de éstos ecosistemas del norte de los Andes, los cuales parecen poseer la capacidad de poner a prueba muchos paradigmas de la ecología, aumentando así la incertidumbre acerca de lo que sería su respuesta al cambio climático. Según este 
estudio, los bosques montanos de los Andes, podrían incluso servir como refugio de la flora y el carbono, asumiendo eso sí, que debido al área total que cubren, éstos no podrían contrarrestar las pérdidas totales en magnitud que eventualmente sufriera su contraparte de tierras bajas. Las acciones encaminadas a la conservación y al entendimiento de la dinámica de estos ecosistemas comprenden la declaratoria de la zona como Área de Reserva Natural y al avance de la investigación sobre la autoecología de muchas de las especies encontradas en la zona con el fin de preparar protocolos de Restauración Ecológica en áreas degradadas de la Reserva, que permitan avanzar en el conocimiento de estas prácticas y a su vez posibiliten su conservación y recuperación.

\section{AGRADECIMIENTOS}

A Darío Sánchez S. por su colaboración en el montaje de las parcelas e identificación de material vegetal. A Jorge Andrés Pérez por su aporte en la remedición de parcelas e identificación de material botánico. A Jesús María Marín por permitir el establecimiento y monitoreo de las parcelas permanentes de investigación.

\section{RESUMEN}

Los bosques sub-Andinos poseen alta diversidad biológica, de los cuales poco se conoce sobre su dinámica natural. Se evaluaron patrones de diversidad y biomasa, tasas de mortalidad y reclutamiento en dos parcelas permanentes de una hectárea cada una, establecidas en bosques en altitudes entre los $2000-2$ 200msnm, en la vertiente Oriental de la cordillera Occidental del norte de los Andes en Colombia. Se determinó diversidad de especies mediante el índice alpha de Fisher, la dinámica del bosque se evaluó mediante tres censos durante nueve años. En total, se hallaron 1964 individuos con un diámetro a la altura del pecho DAP $\geq 10 \mathrm{~cm}$. pertenecientes a 222 especies, 113 géneros y 60 familias. La riqueza media de especies fue de 156 especies por hectárea y un índice alpha medio de Fisher de 56.2/h. Las tasas de mortalidad y reclutamiento fueron de $0.88 \%$ y $1.16 \%$ respectivamente, lo cual no indica que se presente un efecto externo proveniente del calentamiento global sobre la dinámica del bosque. La biomasa aérea promedio fue de $243.44 \pm 9.82 \mathrm{tn} / \mathrm{ha}$ con un incremento anual promedio de 2.9 ton/ha, valor superior a los reportados, lo cual sugiere que el equilibrio para este bosque no se ha alcanzado. De acuerdo con las observaciones de campo, este bosque parece estar recuperándose de una alteración pasada.

Palabras clave: biomasa aérea, mortalidad, reclutamiento, cambio climático, bosques andinos.

\section{REFERENCIAS}

BirdLife International y Conservation InternationalBoyla, K. \& A. Estrada. 2005. Áreas importantes para la C conservación de las aves en los Andes Tropicales: sitios prioritarios para la conservación de la biodiversidad. BirdLife Conservation Series No. 14, BirdLife International and Conservation International, Quito, Ecuador. Quito, Ecuador: BirdLife International (series de conservación de BirdLife No. 14: 1-9).

Brown, A.D. \& M. Kappelle. 2001. Introducción a los bosques nublados del neotrópico: una síntesis regional, p. 25-40. In M. Kappelle \& A.D. Brown (eds.). Bosques nublados del neotrópico. Instituto Nacional de Biodiversidad (INBIO), Santo Domingo de Heredia, Costa Rica.

Cantillo, E.E \&. J.O. Rangel-Ch. 2008. Aspectos de la estructura y del patrón de riqueza de la vegetación del transecto Sumapaz, p. 529-564. In T. Van der Hammen \& A. Cleef (eds.). La Cordillera Oriental (Colombia). Transecto Sumapaz. Estudio de ecosistemas Tropandinos-Ecoandes.

Cascante-Marín, A., V. Meza-Picado \& A. Estrada-Chavarría. 2011. Tree turnover in a premontane tropical forest (1998-2009) in Costa Rica. Plant Ecol. 212: $1101-1108$

Chave, J., R. Condit, H.C. Muller-Landau, S.C. Thomas, P.S. Ashton, S. Bunyavejchewin, L.L. Co, H.S. Dattaraja, S.J. Davies, S. Esufali, C.E.N. Ewango, K.J. Feeley, R.B. Foster, N. Gunatilleke, S. Gunatilleke, P. Hall, T.B. Hart, C. Hernández, S.P. Hubbell, A. Itoh, S. Kiratiprayoon, J.V. LaFrankie, S.L. de Lao, J.R. Makana, Md.N. upardi Noor, A. Rahman-Kassim, C. Samper, R. Sukumar, H.S. Suresh, S. Tan, J. Thompson, M.D.C. Tongco, R. Valencia, M. Vallejo, G. Villa, T. Yamakura, J.K. Zimmerman \& E.C Losos. 2008. Assessing evidence for a pervasive alteration in tropical tree communities. PLoS Biol. 6: 455-462.

Chaves, M.E. \& M. Santamaría. 2006. Informe nacional sobre el avance en el conocimiento y la información de la biodiversidad 1998-2004. Instituto de Investigación de Recursos Biológicos Alexander von Humboldt, Bogotá D.C., Colombia. 
Colwell, R.K., G. Brehm, C.L. Cardelús, A.C. Gilman \& J.T. Longino. 2008. Global warming, elevational range shifts, and lowland biotic attrition in the wet tropics. Science 322: 258-261.

Condit, R., S.P. Hubbell, J.V. Lafrankie, R. Sukumar, N. Manokaran, R. Foster \& P. Ashton. 1996. SpeciesArea and Species-Individual relationships for tropical trees: A comparison of three 50-ha plots. J. Ecol. 4: 549-562.

Condit, R., S. Aguilar, A. Hernandez, R. Pérez, S.L. de Lao, G. Angehr, S.P. Hubbell \& R.B. Foster. 2004a. Tropical Forest dynamics across a rainfall gradient and the impact of an El Niño dry season. J. Trop. Ecol. 20: 51-72.

Condit, R., L. Giles Jr., S.L. de Lao, M.S. Ashton, N.V.L. Brokaw, R. Bunyavejchwin, G.B. Chuyong, L.L. Co, H.S. Dattaraja, S.J. Davies, S. Esufali, C.E.N. Ewango, R.B. Foster, N. Gunatilleke, S. Gunatilleke, T.H. Hart, C. Hernandez, S.P. Hubbell, A. Itoh, R. John, M. Kanzaki, D. Kenfack, S. Kiratiprayoon, J.V. LaFrankie, H.S. Lee, I. Liengola, J.R. Makana, N. Manokaran, M. Navarette Hernandez, T. Ohkugo, R. Perez, N. Pongpattananurak, C. Samper, K. Sri-ngernyuang, R. Sukumar, I.F. Fun, H.S. Sureh, S. Tan, D.W. Thomas, J.D. Thompson, M.I. Vallejo, G.V. Munoz, R. Valencia, T. Yamakura \& J. K. Zimmerman. 2004b. Species-area relationships and diversity measures in the forest dynamics plots, p. 79-89. In E.C. Losos \& E.G. Leigh (eds.). Tropical forest diversity and dynamism: Findings from a large-scale plot network. University of Chicago, Chicago, EEUU.

Condit, R., P. Ashton, S. Bunyavejchewin, H. Dattaraja, S. Davies, S. Esufali, C. Ewango, R. Foster, I. Gunatilleke, C. Gunatilleke, P. Hall, K. Harms, T. Hart, C. Hernandez, S. Hubbell, A. Itoh, S. Kiratiprayoon, J. Lafrankie, S.L. de Lao, M. Loo, N. Jean-Remy, K.M.D. Nur Supardi, R. Abdul, S. Russo, R. Sukumar, C. Samper, H. Suresh, S. Tan, T.S. Homas, R. Valencia, M. Vallejo, G. Villa \& T. Zillio. 2006. The importance of demographic niches to tree diversity. Science 313: 98-101.

Cuadros, T. 1997. Aspectos faunísticos. Reserva natural Farallones del Citará, p. 129-145. In Estudios biofísicos y socioeconómicos. Informe técnico. Universidad Nacional de Colombia, Sede Medellín, Colombia.

Espinal, L.S., J. Tosi, E. Montenegro, G. Toro \& D. Diazgranados. 1977. Mapa ecológico de Colombia, p. 201. Planchas 1:500.000 (21 planchas). Instituto Geográfico Agustín Codazzi, Bogotá, Colombia.

Gentry, A.H. 1982. Patterns of neotropical plant species diversity, p. 1-84. In M.K. Hecht, B. Wallace \& E.T.
Prance (eds.). Evolutionary biology, Plenum, Nueva York, EEUU.

Gentry, A.H. 1995. Patterns of diversity and floristic composition in Neotropical Montane Forests, p. 103-126. In S. Churchill, H. Balslev, E. Forero \& J. Luteyn (eds.). Biodiversity and Conservation of Neotropical Montane Forests. The New York Botanical Garden, Bronx, EEUU.

Homeier, J., S.W. Breckle, S. Gunter, R.T. Rollenbeck \& C. Leuschner. 2010. Tree diversity, forest structure and productivity along altitudinal and topographical gradients in a species-rich Ecuadorian montane rain forest. Biotropica 42: 140-148.

Eggleston, H.S., L. Buendia, K. Miwa, T. Ngara \& K. Tanabe. 2006. IPCC Guidelines for national greenhouse gas inventories. National greenhouse gas inventories programm, IGES, Japón.

Rangel-Ch, J.O., A.M. Cleef, S. Salamanca \& C. Ariza. 2005. La vegetación de los bosques y selvas del Tatamá, p. 459-643. In T. Van der Hammen, J.O. RangelCh \& A.M. Cleef (eds.). La Cordillera Occidental colombiana - Transecto de Tatamá. Estudios de Ecosistemas Tropandinos, J. Cramer, Berlin-Stuttgart, Alemania.

López, W. \& A.J. Duque. 2010. Patrones de diversidad alfa en tres fragmentos de bosques montanos en la región norte de los Andes, Colombia. Rev. Biol. Trop. 58: 483-498.

McCain, C.M. 2004. The mid-domain effect applied to elevational gradients: species richness of small mammals in Costa Rica. J. Biogeogr. 31: 19-31.

Myers, N., R.A. Mittermeier, C.G. Mittermeier, G.A.B. da Fonseca \& J. Kent. 2000. Biodiversity hotspots for conservation priorities. Nature 403: 853-858.

Parra, N. 1997. Geología y geomorfología de los Farallones del Citará, p. 64-86. In Estudios biofísicos y socioeconómicos. Informe técnico. Universidad Nacional de Colombia, Sede Medellín, Colombia.

Phillips, O.L., Y. Malhi, N. Higuchi, W.F. Laurence, P.V. Nuñez, R.M. Vásquez, S.G. Laurence, L.V. Ferreira, M. Stern, S. Brown \& J. Grace. 1998. Changes in the carbon balance of tropical forests: evidence from long-term plots. Science 282: 439-442.

Phillips, O., T.R. Baker, L. Arroyo, N. Higuchi, T. Killeen, W.F. Laurance, S.L. Lewis, J. Lloyd, Y. Malhi, A. Monteagudo, D. Neill, P. Núñez-Vargas, N. Silva, J. Terborgh, R. Vásquez-Martínez, M. Alexiades, S. Almeida, S. Brown, J. Chave, J.A. Comiskey, C.I. Czimczik, A. Di Fiore, T. Erwin, C. Kuebler, S.G. 
Laurance, H.E.M. Nascimento, W. Palacios, S. Patiño, N. Pitman, J. Olivier, C.A. Quesada, M. Saldias, A. Torres-Lezama \& B. Vinceti. 2004. Pattern and process in Amazon tree turnover, 1976-2001. Philos. Trans. R. Soc., B. 359: 381-407.

Phillips, O.L., L. Aragão \& S. Lewis. 2009. Drought Sensitivity of the Amazon Rainforest. Science 323: 1344-1347.

Rodríguez, N., D. Armenteras, M. Morales \& M. Romero. 2004. Ecosistemas de los Andes Colombianos. Instituto de Investigación de Recursos Biológicos Alexander von Humboldt, Bogotá, Colombia.

Rosenzweig, M.L. 1995. Species diversity in space and time. Cambridge University, Cambridge, United Kingdom.

Ruokolainen, K., H. Tuomisto, J. Vormisto \& N. Pitman. 2002. Two biases in estimating range sizes of Amazonian plant species. J. Trop. Ecol. 18: 935-942.

Sánchez, D. \& J.O. Velásquez. 1997. Vegetación y ecología de los Farallones del Citará, p. 101-128. In Estudios biofísicos y socioeconómicos. Informe técnico.
Universidad Nacional de Colombia, Sede Medellín, Colombia.

Sierra, C.A., J.I. del Valle, S.A. Orrego, F.H. Moreno, M.E. Harmon, M. Zapata, G.J. Colorado, M.A. Herrera, W. Lara, D.E. Restrepo, L.M. Berrouet, L.M. Loaiza \& J.F. Benjumea. 2007. Total carbon stocks in a tropical forest landscape of the Porce region, Colombia. For. Ecol. Manage. 243: 299-309.

Vanegas, G.L.1997. Reserva natural Farallones del Citará. Suelos, p.83-87. In Estudios biofísicos y socioeconómicos. Informe técnico. Universidad Nacional de Colombia, Sede Medellín, Colombia.

Zapata, M., G. Colorado \& J.I. del Valle. 2003. Ecuaciones de biomasa aérea para bosques primarios intervenidos y secundarios, p. 87-120. In S. Orrego, J.I. del Valle \& F. Moreno (eds.). Medición de la captura de carbono en ecosistemas forestales tropicales de Colombia, contribuciones para la mitigación del cambio climático. Universidad Nacional de Colombia Sede de Medellín, Departamento de Ciencias Forestales, Centro Andino para la Economía en el Medio Ambiente, Medellín, Colombia. 